\title{
Signatures of intracrystallite and intercrystallite limitations of charge transport in polythiophenes
}

Kiarash Vakhshouri ${ }^{1}$, Brandon H. Smith ${ }^{1}$,Edwin P. Chan ${ }^{2}$, Chenchen Wang ${ }^{3}$, Alberto Salleo ${ }^{3}$, Cheng Wang $^{4}$, Alexander Hexemer ${ }^{4}$,Enrique D. Gomez ${ }^{1,5^{*}}$

${ }^{1}$ Department of Chemical Engineering, Pennsylvania State University, University Park, PA 16802

${ }^{2}$ Polymers Division, National Institute of Standards and Technology, Gaithersburg, Maryland 20899

${ }^{3}$ Materials Science and Engineering, Stanford University, Stanford, CA 94305

${ }^{4}$ Advanced Light Source, Lawrence Berkeley National Laboratory, Berkeley, CA 94530

${ }^{5}$ Materials Research Institute, Pennsylvania State University, University Park, PA 16802

\section{Supporting Information}

Figure S1 and Figure S2 offer a comparison between the output and transfer characteristics of PBTTT thin-film transistors (TFTs) prepared on untreated versus OTS treated (trimethoxy(octadecyl)silane $\mathrm{SAM}$ layer) $\mathrm{Si} / \mathrm{SiO}_{2}$ surfaces. While device performance is significantly lower on the untreated dielectric from both solvents, the trend of higher charge mobility and source-drain current obtained with films cast from chlorobeneze solutions is consistent. Consequently, no surface treatments were employed during device fabrication to avoid dewetting issues over the large range of processing parameters used in the annealing studies. 

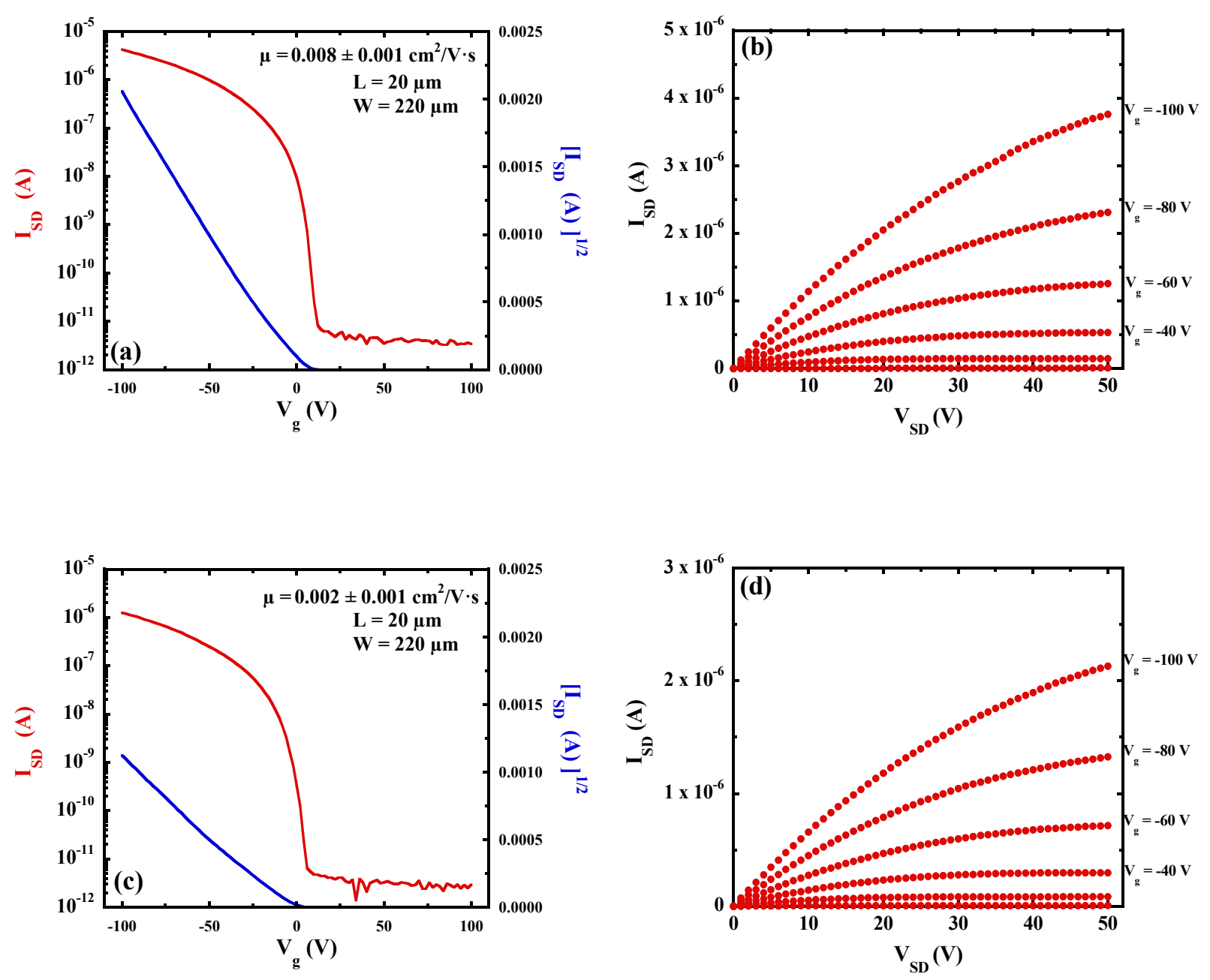

Figure S1. Transfer and output characteristics of PBTTT thin films spun cast from $(a \& b)$ chlorobenzene and annealed at $150^{\circ} \mathrm{C}$ for 10 minutes, and (c \& d) 1,2,4-trichlorobenzene and annealed at $150{ }^{\circ} \mathrm{C}$ for 5 minutes on untreated $\mathrm{SiO}_{2}$ substrates. 

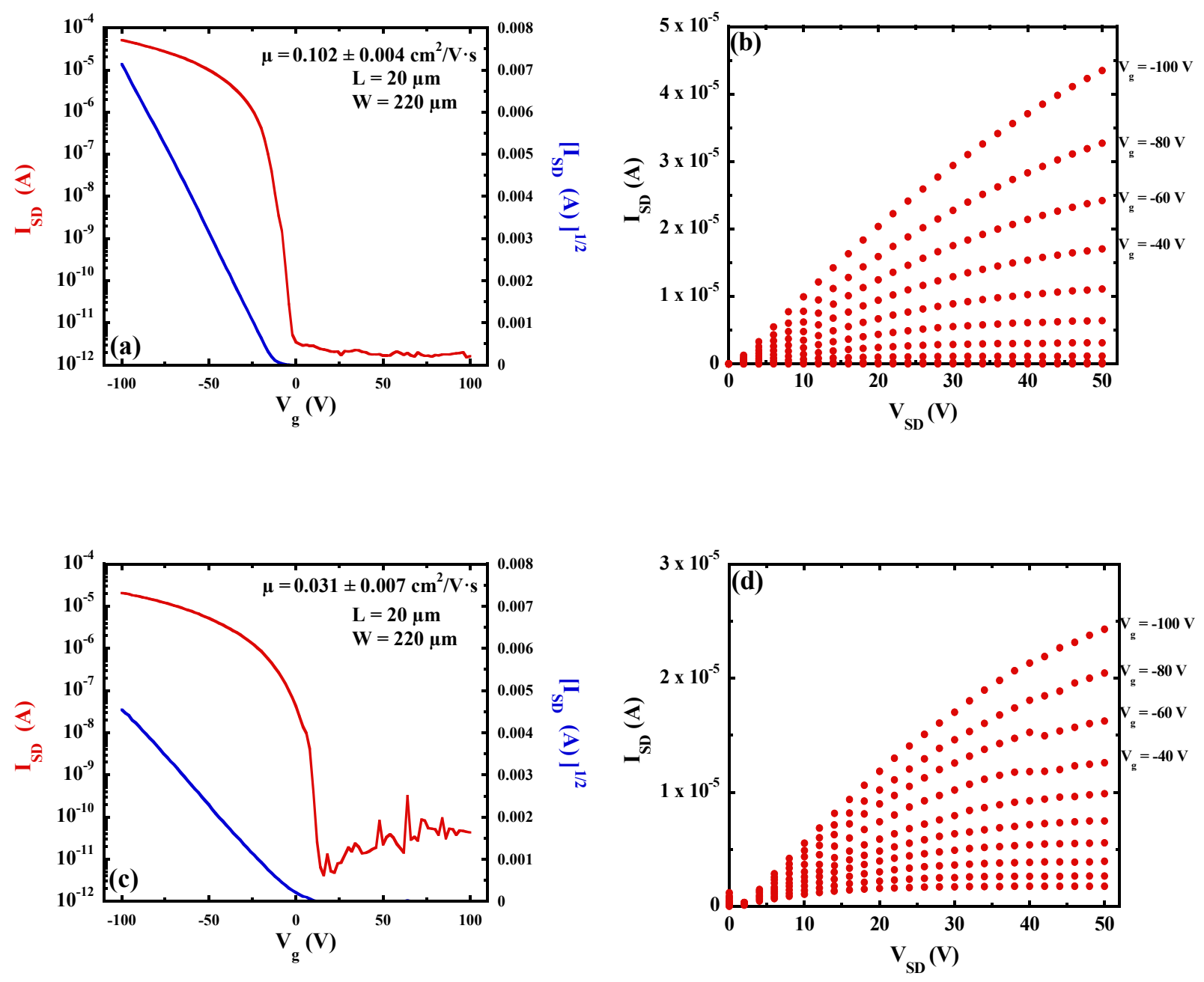

Figure S2. Transfer and output characteristics of PBTTT thin films spun cast from (a \& b) chlorobenzene and annealed at $150{ }^{\circ} \mathrm{C}$ for 10 minutes, and (c \& d) 1,2,4-trichlorobenzene and annealed at $150{ }^{\circ} \mathrm{C}$ for 5 minutes on trimethoxy(octadecyl)silane (OTS) treated $\mathrm{SiO}_{2} \mathrm{substrates}$

Figure S3, S4, S5, and S6 demonstrate the effect of annealing time on crystal orientation and coherence length in the (200) and (010) stacking directions for PBTTT and P3HT films cast from 1,2,4-trichlorobenzene and chlorobenzene. Coherence lengths are calculated using a shape factor $K$ of 0.9 . 

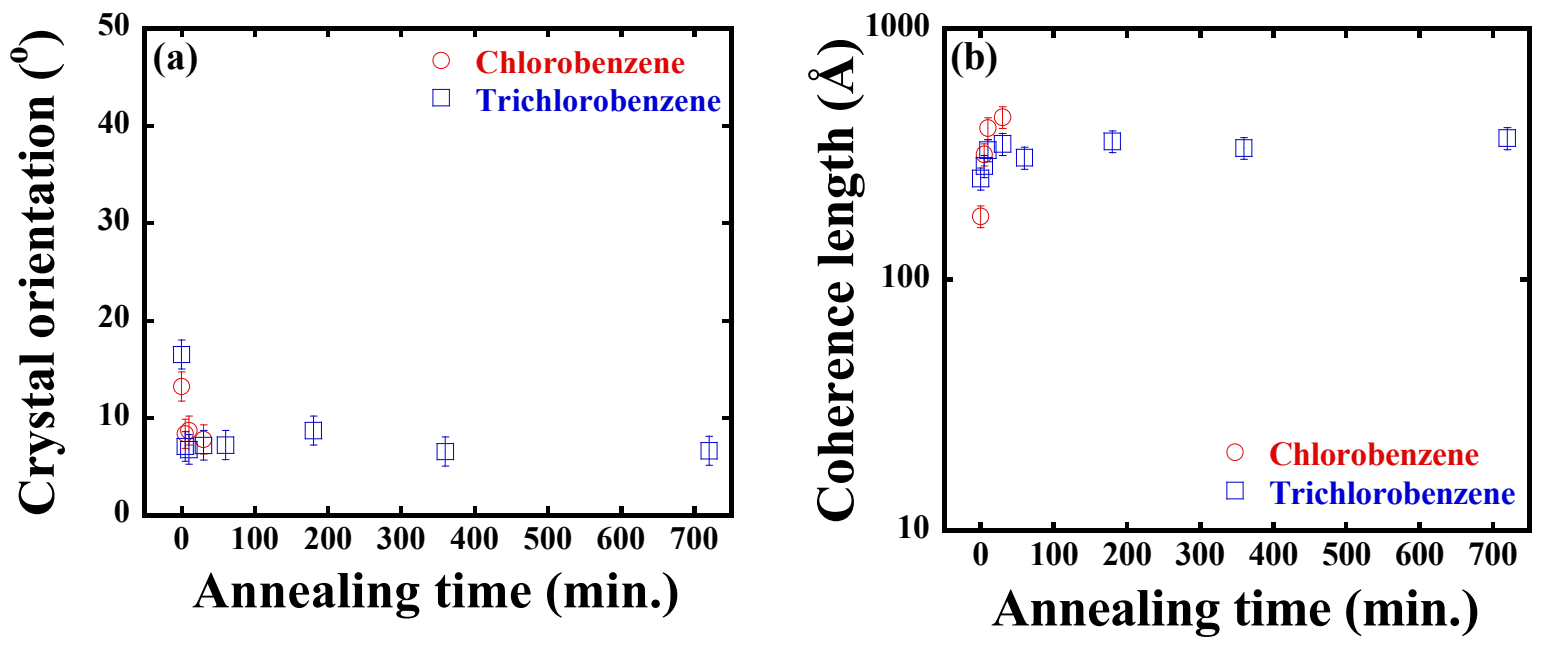

Figure S3. Effect of annealing time on (a) crystal orientation and (b) coherence length for PBTTT thin films spun cast from chlorobenzene and 1,2,4-trichlorobenzene. Samples were annealed at $150^{\circ} \mathrm{C}$. Results are based on the (200) reflection.

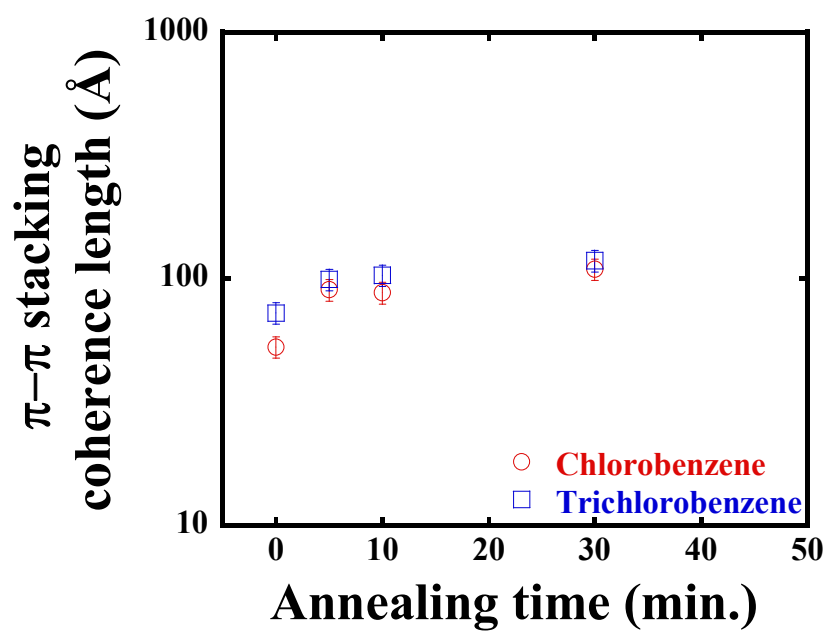

Figure S4. Effect of annealing time on $\pi-\pi$ stacking coherence length for PBTTT thin films spun cast from chlorobenzene and 1,2,4-trichlorobenzene. Samples were annealed at $150{ }^{\circ} \mathrm{C}$. Results are based on the $(010)$ reflection. 

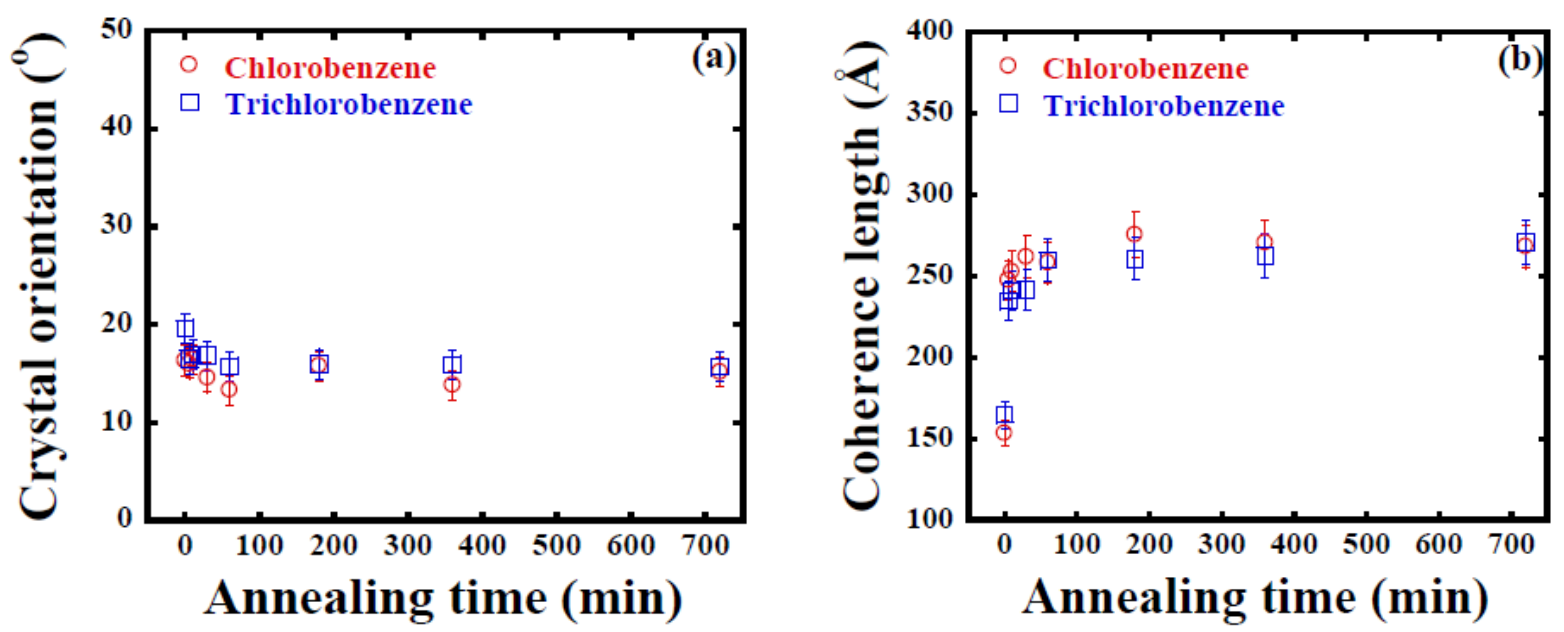

Figure S5. Effect of annealing time on (a) crystal orientation and (b) coherence length for P3HT thin films spun cast from chlorobenzene and 1,2,4-trichlorobenzene. Samples were annealed at $150{ }^{\circ} \mathrm{C}$. Results are based on the (200) reflection.

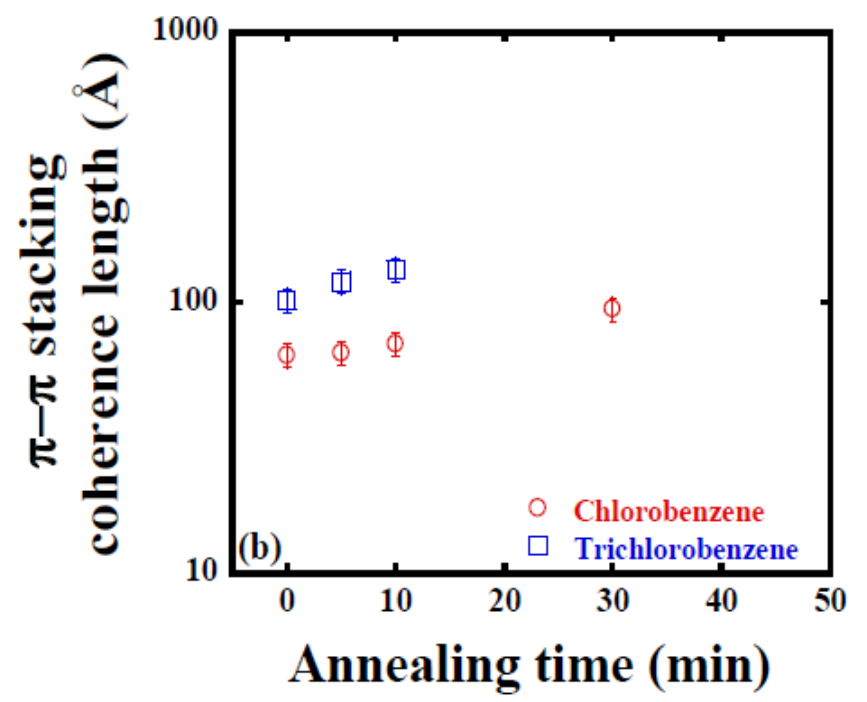

Figure S6. Effect of annealing time on $\pi-\pi$ stacking coherence length for P3HT thin films spun cast from chlorobenzene and 1,2,4-trichlorobenzene. Samples were annealed at $150{ }^{\circ} \mathrm{C}$. Results are based on the (010) reflection. 
Figure S7 contains atomic force microscopy (AFM) micrographs of P3HT thin films cast from 1,2,4-trichlorobenzene and chlorobenzene. While both samples possess equivalent degrees of crystallinity, each film exhibits significant differences in microstructure. Shorter range order is apparent in the film spin cast from chlorobenzene, but enhanced longer range order is displayed in the film prepared from the higher boiling point solvent.
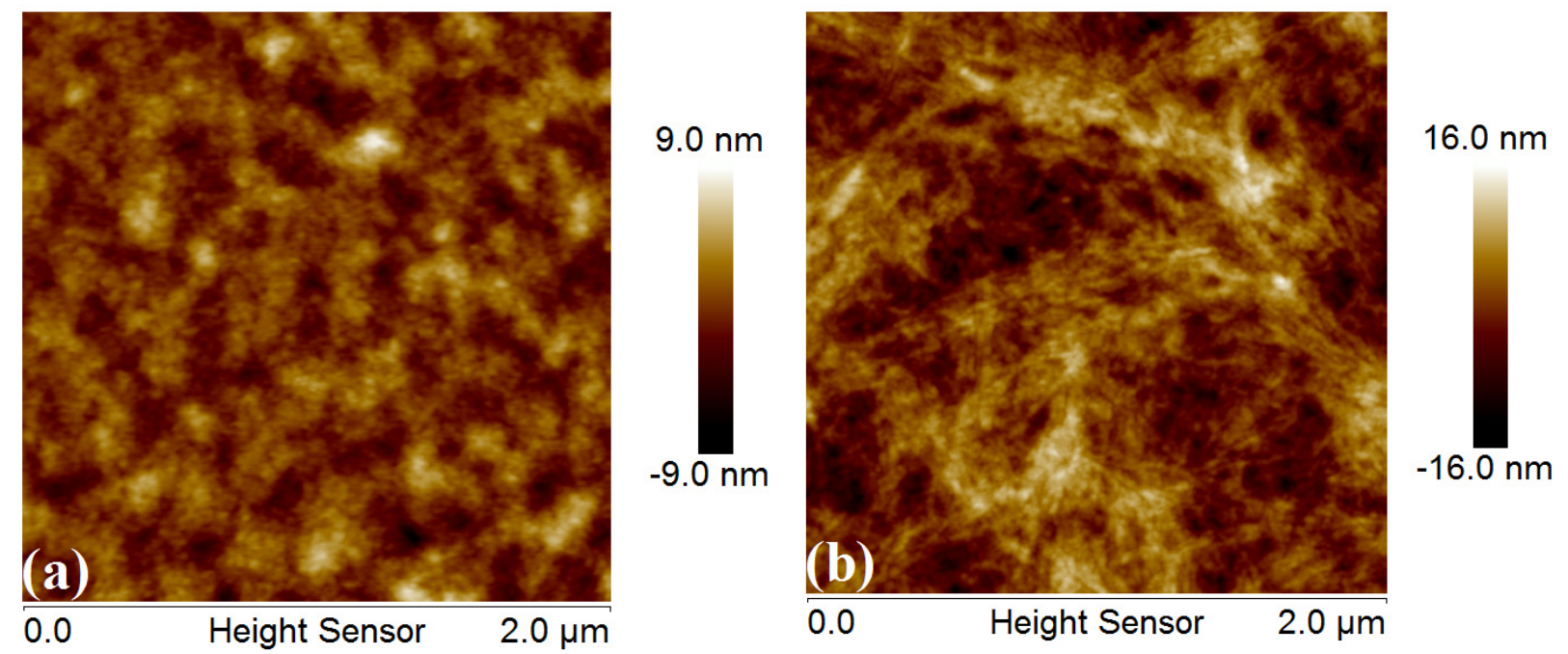

Figure S7. AFM height images of P3HT thin films spun from (a) chlorobenzene and annealed at $150{ }^{\circ} \mathrm{C}$ for 7 minutes and (b) as-cast trichlorobenzene. Both samples have the same degree of crystallinity. 
P-SoXS scattering patterns and radial integrations are included as Figure S8 and Figure S9. The scattering patterns were obtained using linear polarized photons at an energy of $285.4 \mathrm{eV}$. The results confirm that PBTTT films spun cast from chlorobenzene and 1,2,4-trichlorobenzene are isotropic.
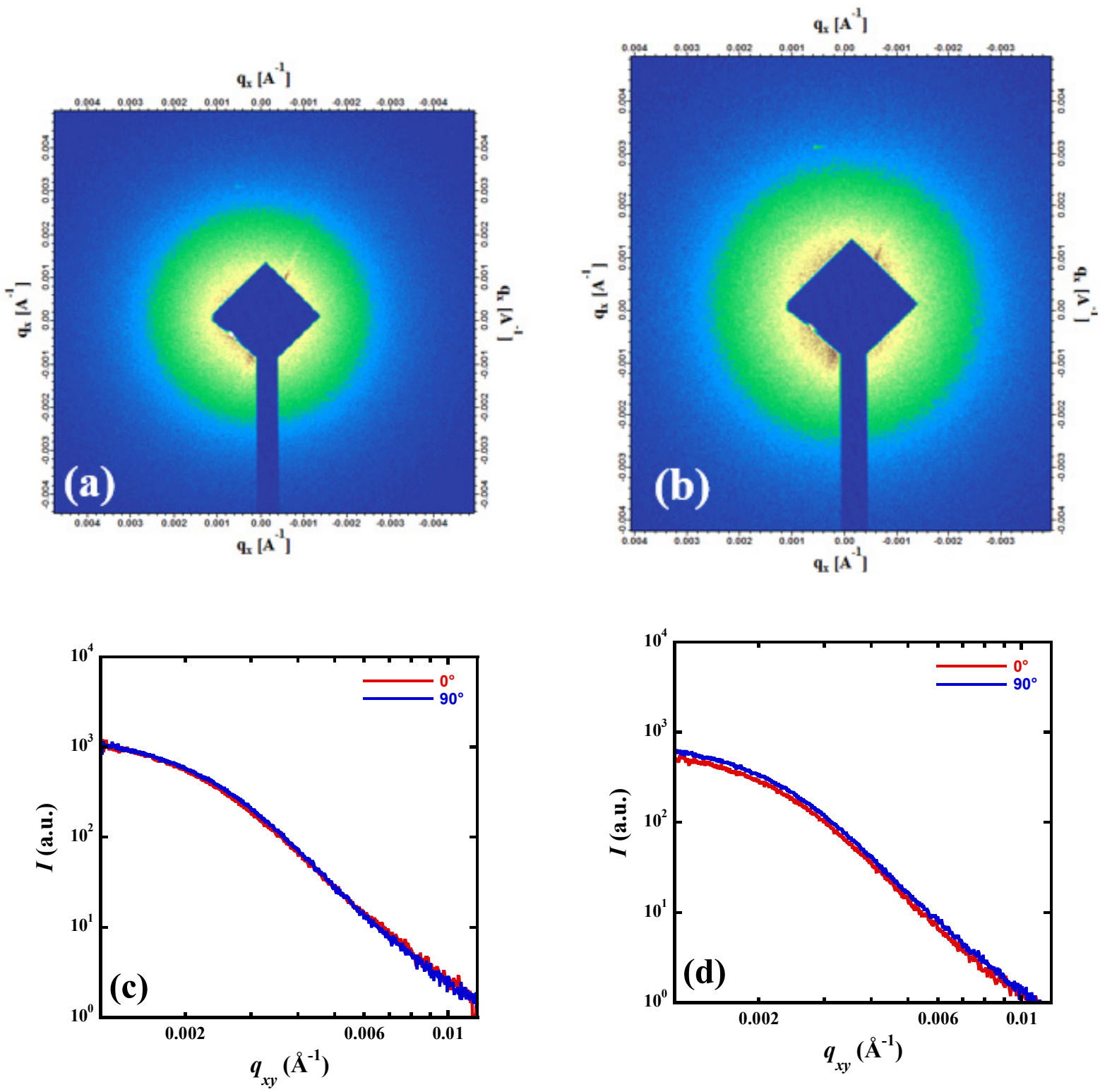

Figure S8. Isotropic scattering patterns obtained using linear (a) horizontally and (b) vertically polarized photons at an energy of $285.4 \mathrm{eV}$ for a thin PBTTT film spin cast from chlorobenzene and annealed at $150{ }^{\circ} \mathrm{C}$ for 10 minutes, and (c \& d) corresponding radial scattering profiles at 0 and $90^{\circ}$. 

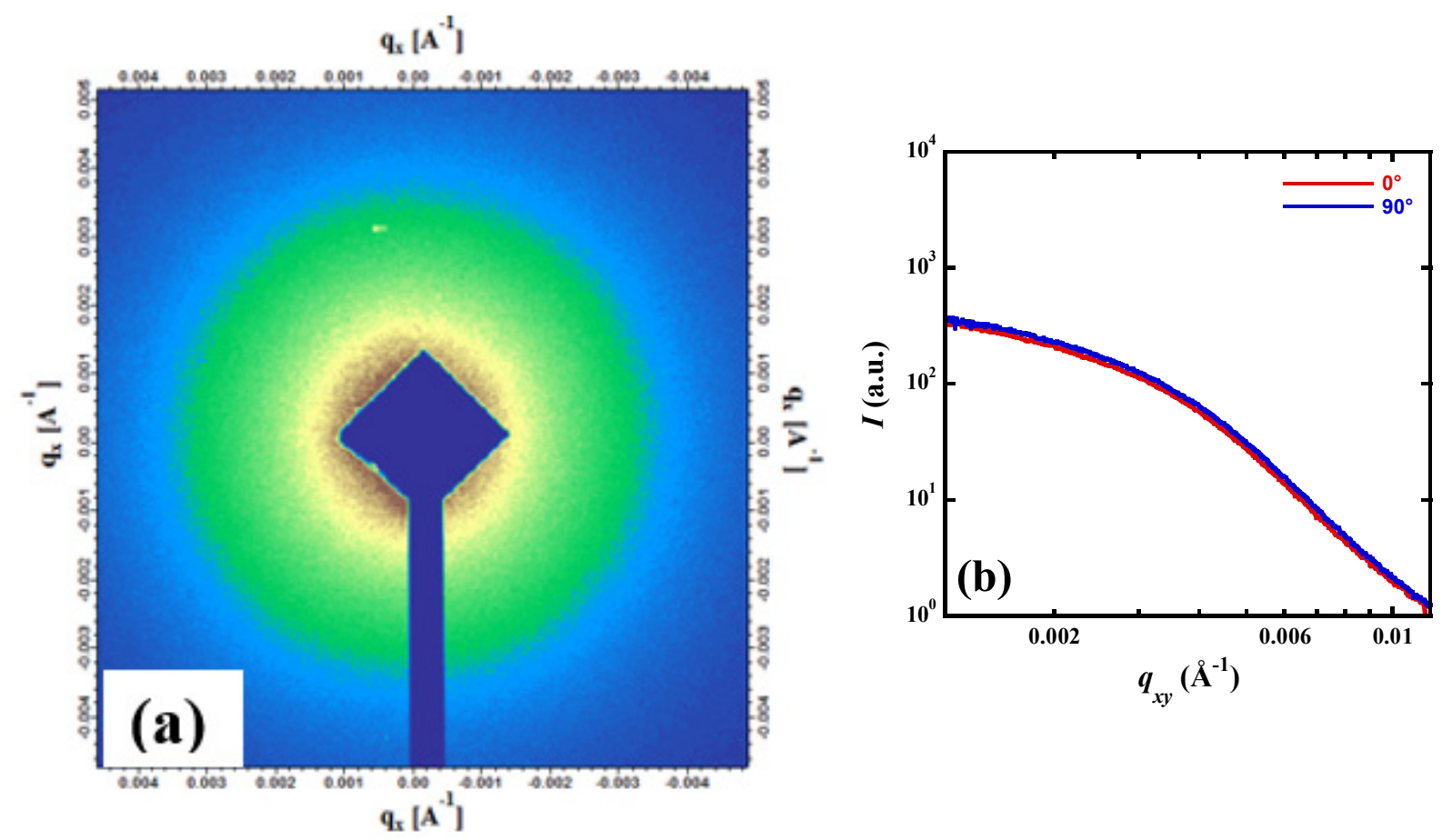

Figure S9. Isotropic scattering pattern (a) and corresponding radial scattering profile (b) at $0^{\circ}$ and $90^{\circ}$ obtained using linear polarized photons at an energy of $285.4 \mathrm{eV}$ for a thin PBTTT film spin cast from 1,2,4-trichlorobenzene and annealed at $150{ }^{\circ} \mathrm{C}$ for 5 minutes.

Figure S10 shows the RSOXS scattering intensity for PBTTT films cast from chlorobenzene and 1,2,4-trichlorobenzene and annealed at $150{ }^{\circ} \mathrm{C}$ for $10 \mathrm{~min}$ and $5 \mathrm{~min}$, respectively. The intensity is shown as $I q^{2}$ to highlight features near scattering vectors of 0.002 and $0.003 \AA^{-1}$. 


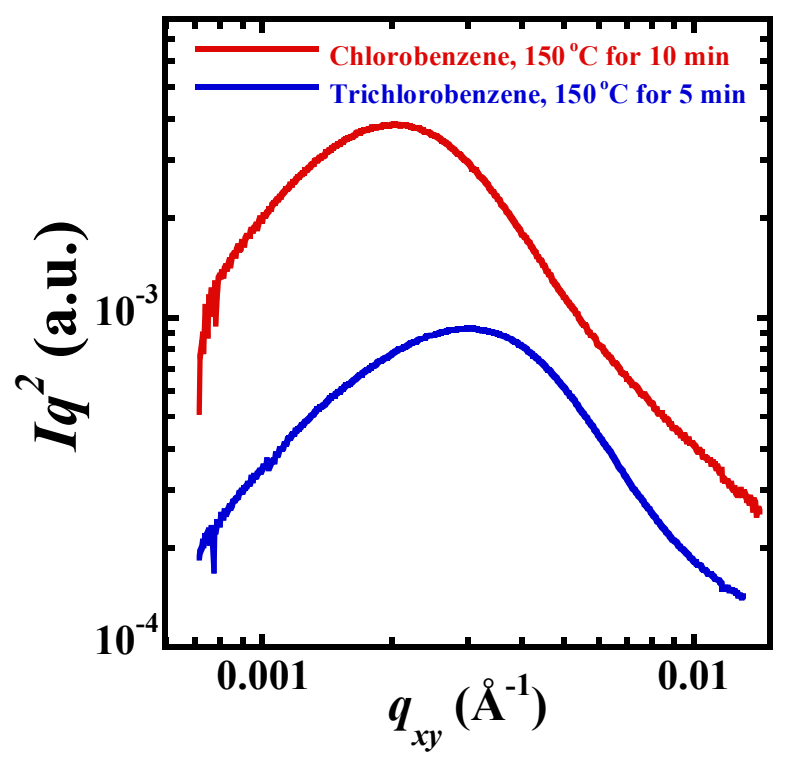

Figure S10. RSOXS intensity versus scattering vector as $I q^{2}$ for PBTTT films cast from chlorobenzene and 1,2,4-trichlorobenzene and annealed at $150{ }^{\circ} \mathrm{C}$ for 10 and $5 \mathrm{~min}$, respectively. Peaks are apparent near 0.002 and $0.003 \AA^{-1}$.

Figure S11 shows the thin-film modulus as a function of strain of P3HT films cast from chlorobenzene and 1,2,4-trichlorobenzene. The casting and annealing conditions are chosen such that the crystallinities are the same for the two films. Although the modulus appears to be slightly larger for films cast from chlorobenzene, the charge mobilities of films cast from 1,2,4trichlorobenzene are higher. Thus, for P3HT, a lack of correlation between the thin-film modulus and the charge mobility exists, suggesting that tie chain densities are not the limiting factor for charge transport within high molecular mass P3HT films. 


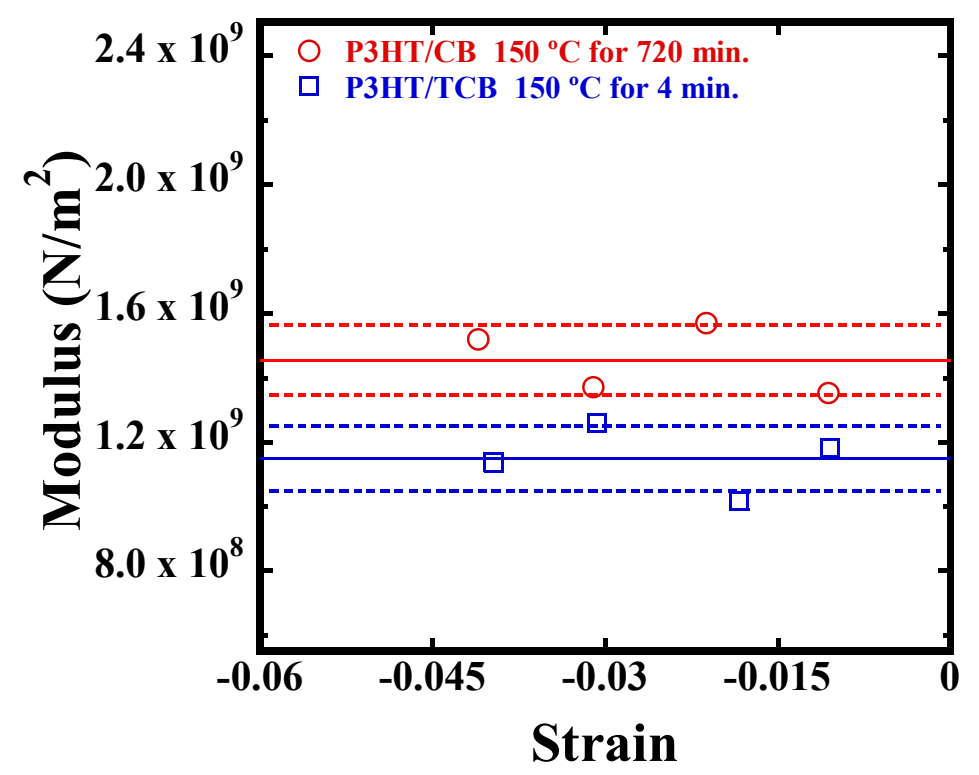

Figure S11. Thin film modulus of P3HT samples spin cast from chlorobenzene and 1,2,4trichlorobenzene and annealed at $150{ }^{\circ} \mathrm{C}$ for 720 and 4 minutes, respectively. Both sets of samples have the same degree of crystallinity. Solid lines represent the average modulus for each case, and dashed lines represent standard deviations. 